\title{
THE KÖLLIKER-FUSE NUCLEUS ACTS AS A TIMEKEEPER FOR LATE-EXPIRATORY ABDOMINAL ACTIVITY
}

\author{
SARAH E. M. JENKIN, ${ }^{a *}$ WILLIAM K. MILSOM $^{a}$ AND \\ DANIEL B. ZOCCAL \\ a Department of Zoology, The University of British Columbia, \\ Vancouver, British Columbia, V6T 1Z4, Canada \\ ${ }^{\mathrm{b}}$ School of Dentistry of Araraquara, São Paulo State \\ University, Araraquara, Brazil
}

\begin{abstract}
While the transition from the inspiratory to the post-inspiratory (post-I) phase is dependent on the pons, little attention has been paid to understanding the role of the pontine respiratory nuclei, specifically the Kölliker-Fuse nucleus (KF), in transitioning from post-I to the late expiratory (late-E) activity seen with elevated respiratory drive. To elucidate this, we used the in situ working heartbrainstem preparation of juvenile male Holtzman rats and recorded from the vagus (cVN), phrenic (PN) and abdominal nerves $(A b N)$ during baseline conditions and during chemoreflex activation [with potassium cyanide (KCN; $n=13)$ or hypercapnia $\left.\left(8 \% \mathrm{CO}_{2} ; n=10\right)\right]$ to recruit active expiration. Chemoreflex activation with KCN increased PN frequency and cVN post-I and $A b N$ activities. The inhibition of KF with isoguvacine microinjections $(10 \mathrm{mM})$ attenuated the typical increase in PN frequency and cVN post-I activity, and amplified the $A b N$ response. During hypercapnia, $A b N$ late-E activity emerged in association with a significant reduction in expiratory time. KF inhibition during hypercapnia significantly decreased PN frequency and reduced the duration and amplitude of post-I cVN activity, while the onset of the AbN late-E bursts occurred significantly earlier. Our data reveal a negative relationship between KF-induced post-I and $A b N$ late-E activities, suggesting that the KF coordinates the transition between post-I to late-E activity during conditions of elevated respiratory drive. (C) 2017 IBRO. Published by Elsevier Ltd. All rights reserved.
\end{abstract}

Keywords: pons, Kölliker-Fuse, active expiration, post-inspiration, hypoxia, hypercapnia.

\section{INTRODUCTION}

Breathing is dependent on multiple motor outputs whose activities are precisely timed to the transition between

\footnotetext{
${ }^{*}$ Corresponding author.

E-mail address: jenkin@zoology.ubc.ca (S. E. M. Jenkin).

Abbreviations: $\mathrm{AbN}$, abdominal nerves; ANOVA, analysis of variance; aug-E, augmenting expiratory; BötC, Bötzinger Complex; CPG, central pattern generator; $\mathrm{cVN}$, vagus nerves; $\mathrm{KCN}$, potassium cyanide; $\mathrm{KF}$, Kölliker-Fuse nucleus; late-E, late expiratory; PN, phrenic nerve; PN, phrenic nerves; post-I, post-inspiratory; preBötC, preBötzinger Complex; VRC, ventral respiratory column.
}

the phases of the breathing cycle. At rest, the mammalian breathing cycle is composed of three phases (Richter, 1982; Smith et al., 2007). The first phase, inspiration (I), is dependent on the active contraction of the inspiratory muscles to draw air into the lungs. During the second phase, post-inspiration (post-I), prolonged inspiratory muscle activity (primarily the diaphragm) and laryngeal muscle contraction counteract the elastic recoil forces of the lungs and chest wall to control the rate that air is exhaled from the lungs (Gautier et al., 1973; Davis and Bureau, 1987; Feldman et al., 2013; Dutschmann et al., 2014). During the third phase (E2), there is no muscle contraction and no airflow (Richter, 1996; Feldman and McCrimmon, 2003). Thus, under eupneic conditions, expiration is considered to be mostly passive (Jenkin and Milsom, 2014).

The three-phase eupneic breathing pattern is the product of the coordinated activity of the respiratory central pattern generator (CPG) located in the brainstem, especially within the ventral respiratory column (VRC) of the medulla (Smith et al., 2007). The current hypothesis is that inspiratory neurons of the preBötzinger Complex (preBötC) generate inspiratory activity, while neurons of the Bötzinger Complex (BötC), essentially post-inspiratory (post-I) and augmenting expiratory (aug-E) neurons, control the expiratory phase (Smith et al., 1991; Richter, 1996; Onimaru and Homma, 2003; Janczewski and Feldman, 2006; Alheid and McCrimmon, 2008; Smith et al., 2009, 2013). The respiratory rhythm generator circuitry is certainly complex, involving degenerate and redundant pontine and medullary regions that can influence respiratory pattern formation (Jones and Dutschmann, 2016). At its simplest level, neurons from the preBötC and BötC establish mutual connections with pontine and medullary respiratory groups, sending projections to cranial and spinal pre-motor and motor nuclei to coordinate the muscle contraction and relaxation cycles that underpin breathing under resting conditions (Smith et al., 2013).

Under conditions of elevated respiratory drive (i.e. hypercapnic or hypoxic conditions), expiration often becomes an active process, and recruitment of the abdominal muscles forcefully exhales air from the lungs (Lemes and Zoccal, 2014; Jenkin and Milsom, 2014). The emergence of active expiration relies on the activation of a conditional active expiratory oscillator located in the ventral medulla, rostral to the VRC, within the retrotrapezoid nucleus/parafacial respiratory group (RTN/ pFRG) (Janczewski and Feldman, 2006). It has been 
hypothesized that the RTN/pFRG provides excitatory inputs to pre-motor expiratory neurons of the VRC, critical for powering the forceful contraction of the abdominal muscles during the late part of the expiratory phase (late-E) (Molkov et al., 2010; Pagliardini et al., 2011; Silva et al., 2016). However, the neural mechanisms responsible for the activation and coordination of the expiratory oscillator with the respiratory CPG remain to be elucidated. Active expiration has been suggested as an important component of the ventilatory response to hypoxia, hypercapnia and exercise (Abraham et al., 2002; Abdala et al., 2009a; Moraes et al., 2012a,b) while abnormal activation of the expiratory oscillator has been associated with the generation of respiratory instabilities of some pathological states, such as Rett syndrome (Abdala et al., 2009b). Therefore, the identification of the neural substrates and their functional role for the control of active expiration has both physiological and pathological relevance.

Excitatory drive from the pons is necessary for generating abdominal muscle burst activity during the late-E phase (Abdala et al., 2009a,b; Molkov et al., 2010). Within the dorsal pons, there is a group of respiratory neurons within the Kölliker-Fuse nucleus (KF), which interact with and modify the output of the medullary areas described above and is critical for the transition from inspiration to expiration (Dutschmann and Herbert, 2006). The $\mathrm{KF}$ is suggested to send excitatory inputs to the VRC neurons, mainly to BötC post-I neurons, which are essential for terminating inspiration, regulating post-I activity in the phrenic and cervical vagus nerves, then controlling the transition from inspiration to expiration (Smith et al., 2007). The KF also provides the drive that excites upper airway musculature contraction during the early (post-I) phase of expiration (Dutschmann and Herbert, 2006). Although anatomical studies have also indicated connections between KF and the ventromedullary areas responsible for the generation of expiratory pattern (Rosin et al., 2006), there is no functional evidence about the role of the pons in regulating the transition from the post-I to the late$E$ phase, or in generating or modifying the transition from a eupneic passive to an active expiratory pattern when drive is elevated. Therefore, it becomes important to investigate the contribution of the KF for the control of late-E abdominal activity, in order to expand our knowledge about the neural circuitry required for the generation of active expiration and the processing of ventilatory responses to hypoxia and hypercapnia. In the present study, we hypothesized that the KF also controls the timing of the phase transition from the post-I to the late-E phase, as well as the onset of late-E activity in abdominal motor nerve output.

\section{EXPERIMENTAL PROCEDURES}

The experimental procedures followed the guidelines of the Brazilian National Council for Animal Experimentation Control (CONCEA) and of the US National Institutes of Health (NIH, publication no. 85-23, 1996). All experimental protocols were authorized by the Local Ethical Committee in Animal Experimentation of the School of Dentistry of Araraquara (protocol 18/2014) and the University of British Columbia (protocol A-130025).

\section{Animals}

Juvenile male Holtzman rats (postnatal day 25-30, 60$80 \mathrm{~g}, n=13$ ) were used in the present study. Animals were housed under controlled conditions of temperature $\left(22 \pm 1^{\circ} \mathrm{C}\right)$ and humidity $(50-60 \%)$ under a 12-h light/dark cycle (lights on at 7:00 am) with free access to rat chow and water.

\section{The in situ working heart brain preparation}

Working heart-brainstem preparations (Paton, 1996) were surgically prepared, as previously described (Zoccal et al., 2008). Juvenile Holtzman rats were deeply anesthetized with halothane until the paw and tail pinch reflexes were abolished. The animals were then transected below the diaphragm and submerged in a cold Ringer solution (in mm: $\mathrm{NaCl}, 125 ; \mathrm{NaHCO}_{3}, 24 ; \mathrm{KCl}, 3$; $\mathrm{CaCl}_{2}, 2.5 ; \mathrm{MgSO}_{4}, 1.25 ; \mathrm{KH}_{2} \mathrm{PO}_{4}, 1.25$; dextrose, 10). Animals were then decerebrated and their skin removed. The lungs were removed with care to not damage the descending aorta or the phrenic nerve at the attachment to the diaphragm. The left cervical vagus nerve was isolated. Preparations were then transferred to a recording chamber; the descending aorta was cannulated and perfused retrogradely $\left(21-25 \mathrm{~mL} \cdot \mathrm{min}^{-1}\right.$; Watson-Marlow 502s, Falmouth, Cornwall, UK), via a double-lumen cannula, with Ringer solution containing $1.25 \%$ Polyethylene glycol (an oncotic agent, Sigma, St Louis, USA) and vecuronium bromide (a neuromuscular blocker, 3$\left.4 \mu \mathrm{g} \cdot \mathrm{mL}^{-1}\right)$. The perfusion pressure was held within 50 $70 \mathrm{mmHg}$ by adding vasopressin (0.6-1.2 nM, Sigma, St. Louis, MO, USA) to the perfusate. The perfusate was continuously gassed with $5 \% \mathrm{CO}_{2}$ and $95 \% \mathrm{O}_{2}$, warmed to $31-32{ }^{\circ} \mathrm{C}$ and filtered using a nylon mesh $(25 \mu \mathrm{m})$.

\section{Nerve recordings and data analyses}

Respiratory motor nerves were isolated and recorded from glass suction bipolar electrodes held in place with micromanipulators. The left phrenic nerve (PN) discharge was recorded from its central end. The left vagus nerve (cVN) was cut at the cervical level (below the carotid artery bifurcation) and its efferent activity was recorded. An abdominal nerve $(\mathrm{AbN})$ was isolated from the abdominal muscles on the right side at the thoracic-lumbar level (T12-L1), cut distally and its activity recorded. All the signals were amplified (P511 AC preamplifier, Grass Instruments, Astro-Med, USA; calibrated prior to the experiments using an internal calibrator), band-pass filtered $(0.3-3 \mathrm{kHz})$ and acquired with an $A / D$ converter to a computer using Spike 2 software (Cambridge Electronic Design, CED, Cambridge, UK).

The analyses of the PN, cVN and $\mathrm{AbN}$ activities were carried out on the rectified and smoothed signals (time constant of $50 \mathrm{~ms}$ ) and performed off-line using Spike 2 
software with custom-written scripts after noise subtraction (as described by Zoccal et al., 2008). PN activity was analysed by its burst amplitude $(\mu \mathrm{V})$, frequency (derived from the time interval between consecutive bursts, expressed as cycles per minute, cpm), duration (time of inspiration, s) and interval (time of expiration, s). For the $\mathrm{cVN}$, activities of inspiratory (coincident with PN burst) and post-inspiratory (remaining activity during the expiratory period) components were measured as the area under the curve $\left(\mu \mathrm{V} \cdot \mathrm{s}^{-1}\right)$ to consider both amplitude and duration. Baseline AbN activity was evaluated as the mean activity $(\mu \mathrm{V})$ during the first (E1 phase) and second half of expiratory periods (E2 phase). The $\mathrm{AbN}$ response to peripheral chemoreflex stimulation was quantified as the mean activity during the total expiratory period $(\mu \mathrm{V})$ whilst hypercapnia-induced $\mathrm{AbN}$ late-E bursts were evaluated by their amplitude $(\mu \mathrm{V})$ and duration relative to PN bursts (s). The respiratory changes elicited by peripheral chemoreflex stimulation and hypercapnia were calculated as the maximal variation relative to the respective baseline. Epochs of 3-4 respiratory cycles (from the initiation of the responses) were considered to calculate the maximal changes to peripheral chemoreflex stimulation while 10-15 cycles (45-60 s) were used to measure the responses to hypercapnia. The magnitudes of the changes were expressed either in their original units or in percentage variation.

\section{Activation of peripheral and central chemoreceptors}

The in situ preparations were exposed to potassium cyanide $(\mathrm{KCN})(n=13)$ or hypercapnia $(n=10)$ to activate peripheral and central chemoreceptors and generate active expiration. Exposure was randomized for each trial. The $\mathrm{KCN}(50 \mu \mathrm{L}, 0.05 \%)$ was injected into the descending aortic via the perfusion cannula. Hypercapnia was achieved by raising the fractional concentration of $\mathrm{CO}_{2}$ in the perfusate from 5 to $8-10 \%$ (balanced with $\mathrm{O}_{2}$ ) for 5-10 min, using a gas-mixing device (AVS Projetos, São Carlos, Brazil) connected to cylinders of pure $\mathrm{O}_{2}$ and $\mathrm{CO}_{2}$. Both stimuli produced consistent respiratory reflex responses, with low variability among preparations.

\section{Microinjections into the KF}

Bilateral microinjections of isoguvacine (a $\mathrm{GABA}_{\mathrm{A}}$ agonist, $10 \mathrm{mM}$, Sigma-Aldrich) containing $5 \%$ fluorescent latex microbeads (Lumafluor, New City, NY, USA) were performed into the KF to pharmacologically inhibit this region. Before the isoguvacine microinjections, the KF was functionally identified by microinjections of L-glutamate (10 mM, Sigma-Aldrich), which evoked an increase in cVN post-I activity and PN apnea (Bautista and Dutschmann, 2014), as demonstrated in Fig. 1. The glass pipettes contained either the drug-microbead mixture or L-glutamate and were positioned in the KF using the following stereotaxic coordinates: $5.5 \mathrm{~mm}$ rostral from the calamus scriptorius; $1.75 \mathrm{~mm}$ lateral from midline; and $1.5 \mathrm{~mm}$ ventral to the dorsal surface. The volume of each microinjection was $70-90 \mathrm{~nL}$. Despite the accuracy of the bilateral injections (as observed with both the histology and the consistent reduction in the cVN post-inspiratory peak), these injections may have not necessarily inhibited all KF neurons.

\section{Experimental Design}

Initially, the functional identification of the KF with Lglutamate microinjections was performed 10-15 min before the beginning of the experimental protocol, as illustrated in Fig. 1. After this period, $\mathrm{KCN}$ or hypercapnia was administrated in order to check the preparation's viability and to obtain baseline responses. A period of at least 10 min was allowed between stimuli. After the preparation had returned to baseline conditions, the effect of KF inhibition on the peripheral chemoreceptor responses and hypercapnic responses was tested. With respect to the peripheral chemoreflex, the injection of KCN was carried out 20-60 s after bilateral microinjections of isoguvacine into the KF. Regarding the hypercapnia response, bilateral isoguvacine microinjections were performed during the exposure to high $\mathrm{CO}_{2}$ levels $\left(8-10 \% \mathrm{CO}_{2}\right)$, usually 3$5 \mathrm{~min}$ after the initiation of hypercapnia. Bilateral microinjections in the KF were performed using a single barrel micropipette and were achieved in under $2 \mathrm{~min}$. The maximal effects were observed for 1-5 min after injections. A total of 13 in situ preparations were included in the data analyses. Ten in situ preparations were exposed to both KCN and hypercapnia, which were administrated randomly and after the recovery from the effects of isoguvacine microinjections in the KF (on average $30 \mathrm{~min}$ ). Three in situ preparations received $\mathrm{KCN}$ only. No differences were noted either in the effects induced by isoguvacine microinjections or the $\mathrm{KCN}$ responses between these 2 subgroups. $\mathrm{PN}, \mathrm{AbN}$ and cVN activities were recorded simultaneously in all in situ preparations with exception of one preparation, in which cVN activity was not recorded.

\section{Histology}

At the end of each experiment, the brain stem was removed and fixed by immersion for 5 days in $10 \%$ buffered formalin and then in sucrose solution (20\%) overnight. After, $40 \mu \mathrm{m}$ coronal sections were obtained at the level of KF (Leica, CM1850 UV; Wetzlar, Hesse, Germany) and analysed in a fluorescent microscope (Leica, DM5500 B) with the aid of a rat brain Atlas (Paxinos and Watson, 2009) to identify the injections sites (fluorescent microbeads; Fig. 2).

\section{Statistical analysis}

All results are expressed as means \pm standard errors of the means, and comparison between conditions was done with repeated measures ANOVA. If normality was not passed, a non-parametric repeated measures ANOVA alternative, the Friedman test, was performed. This test provides a chi-square statistic for ranks. HolmSidak and Tukey post hoc tests were used as appropriate. The comparisons were conducted with 


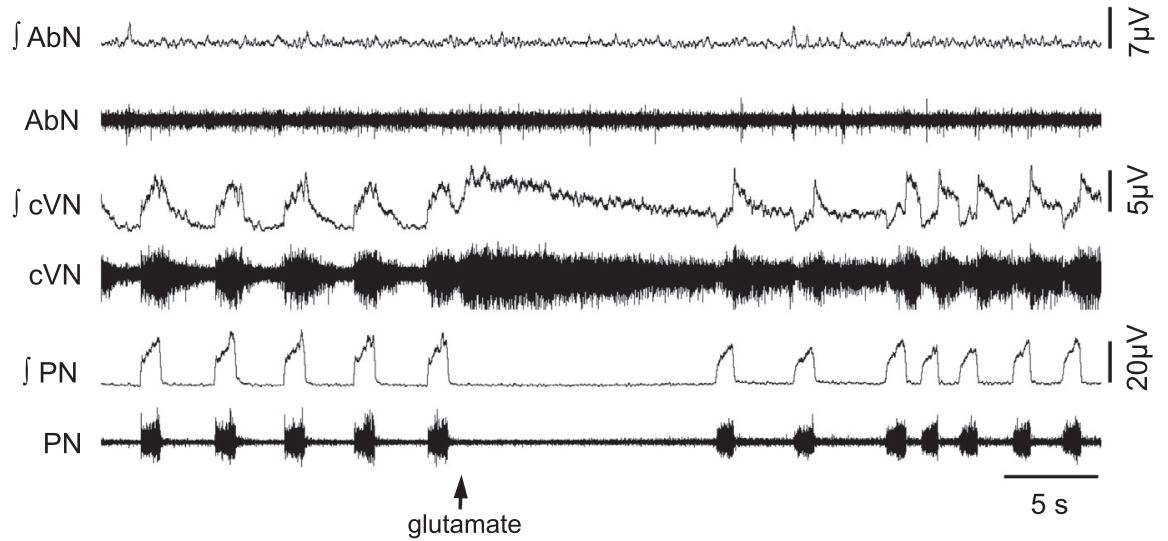

Fig. 1. Raw and integrated $\left(\int\right)$ recordings of abdominal $(A b N)$, cervical vagus $(c V N)$ and phrenic nerve (PN) activities of an in situ preparation, representative from the group, illustrating the pattern of respiratory changes elicited by unilateral microinjection of glutamate into the KF (arrow).
A

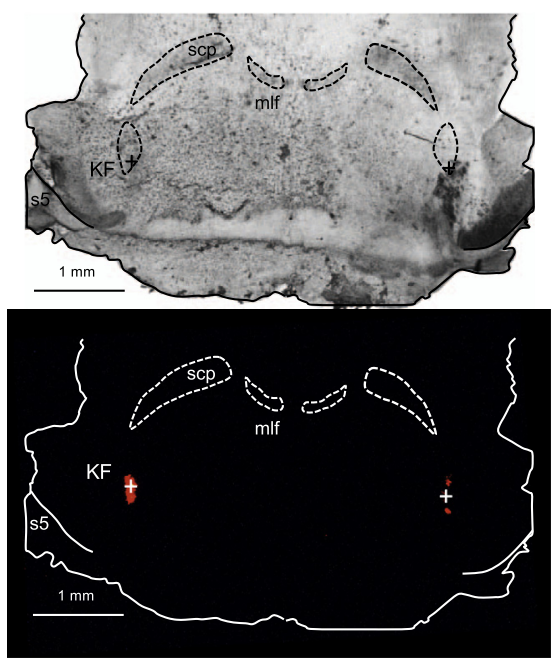

B

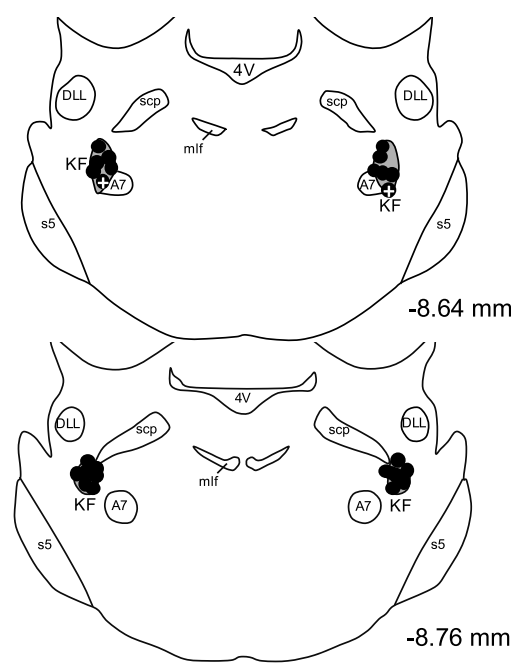

Fig. 2. Panel A: Bright-field (top) and fluorescent (bottom) microscope images of brainstem coronal sections of a representative in situ preparation, showing the injection sites of isoguvacine into the KF (indicated with the crosses). Panel B: schematic representation of all injection ( $n=13$, black circles) sites into the KF. The crosses represent the injection sites of isoguvacine into the KF. Abbreviations: 4V - 4th ventricle; DLL - dorsal nucleus of the lateral lemniscus; mlf medial longitudinal fasciculus; scp - superior cerebellar peduncle; s5 - sensory root of trigeminal nerve.

Sigma Stat 11 software (Dundas Software LTD) and differences were considered significant when $p<0.05$. isoguvacine on baseline respiratory related neural output. After bilateral inhibition of the KF with isoguvacine ( $n=13$ ) during baseline conditions (Fig. 3A, B) there was no significant change in $\mathrm{PN}$ frequency (14.81 \pm 1.24 vs. $11.98 \pm 1.13 \mathrm{bpm}, \chi^{2}(1$ $n=13)=0.69, p=0.581$, Fig. 3B), but there was a significant decrease in PN amplitude (12.56 \pm 1.59 vs. $9.91 \pm 1.18 \mu \mathrm{V}, \quad \chi^{2}(1, \quad n=13)$ =9.31, $\quad p=0.003$, Fig. 3C). Moreover, isoguvacine microinjections in the KF significantly increased inspiratory time (1.07 \pm 0.05 vs. $1.76 \pm 0.15 \mathrm{~s}, \quad F(1,12)$ $=19.55, \quad p<0.001$, Fig. 3D), but had no effect on the expiratory time $(3.57 \pm 0.40$ vs. $4.04 \pm 0.46 \mathrm{~s}, \quad F$ $(1,12)=0.817 p=0.384$, Fig. 3E). Bilateral inhibition of the KF significantly increased cVN inspiratory activity $\quad(8.56 \pm 0.77 \quad$ vs. $\quad 11.86$ $\pm 1.11 \mu \mathrm{V} \cdot \mathrm{s}, \quad F(1,11)=21.00$, $p<0.001$, Fig. 3F) and significantly decreased cVN post-I area under the curve $\quad(16.78 \pm 3.22 \quad$ vs. 7564 $\pm 1.72 \mu \mathrm{V} \cdot \mathrm{s}, \quad \chi^{2}(1, \quad n=12)=12.00$, $p<0.001$; $\quad$ Fig. 3G). Bilateral inhibition of the KF had no significant effect on $\mathrm{AbN}$ mean activity during $\mathrm{E} 1$ $(2.31 \pm 0.59 \quad$ vs. $2.21 \pm 0.64 \mu \mathrm{V}$, $\chi^{2}(1, \quad n=13)=0.077, \quad p=1.000$, Fig. $3 \mathrm{H})$ or during E2 $(3.93 \pm 1.24$ vs. $4.27 \pm 1.37 \mu \mathrm{V}, \chi^{2}(1, n=13)=3.77$, $p=0.092$, Fig. 3I). In all animals included in the analyses presented the microinjections sites were within the boundaries of the KF (Fig. 2), extending from -8.64 to $-8.76 \mathrm{~mm}$ in relation to bregma (Paxinos and Watson, 1998). Despite the accuracy of the bilateral injections (as observed with both the histology and the consistent reduction in the cVN postinspiratory peak), these injections may have not necessarily inhibited all KF neurons.

\section{RESULTS}

Under basal conditions $\left(95 \% \mathrm{O}_{2}\right.$ and $\left.5 \% \mathrm{CO}_{2}\right)$ all in situ preparations exhibited a eupneic-like breathing pattern, with ramping $\mathrm{PN}$ bursts, biphasic cVN activity consisting of an inspiratory component followed by decrementing post-I activity, and low-amplitude AbN activity (Fig. 3A).

\section{Effect of isoguvacine microinjections in the KF on baseline activities}

Fig. 3A shows a representative trace demonstrating the effect of inhibiting the KF with bilateral injections of

\section{Effect of KF inhibition with isoguvacine on the peripheral $\mathrm{O}_{2}$ chemoreflex activation}

Stimulation of the peripheral chemoreceptors with $\mathrm{KCN}$ before inhibition of the KF $(n=13$, Fig. 4A, B) elicited a transient tachypneic response $(14.39 \pm 1.01$ vs. 22.72 $\pm 1.42 \mathrm{bpm}, \quad F(3,36)=\quad 11.28, \quad p<0.001$; Fig. $4 A, 4 B$ and $4 C)$, with significant increases in $P N$ burst amplitude ( $\triangle \mathrm{PN}$ amp: $19.17 \pm 4.39 \%, \quad \chi^{2}(1$, $n=13)=13.00, \quad p<0.001$, Fig. 4D). $\mathrm{KCN}$ also increased both the $\mathrm{cVN}$ inspiratory $(\Delta: 14.34 \pm 1.68 \%$, $\chi^{2}(1, n=12)=12.00, p<0.001$, Fig. 4E) and post-I activities $\quad(\Delta: \quad 26.20 \pm 4.19 \%, \quad F(1,11)=38.42$, 
A

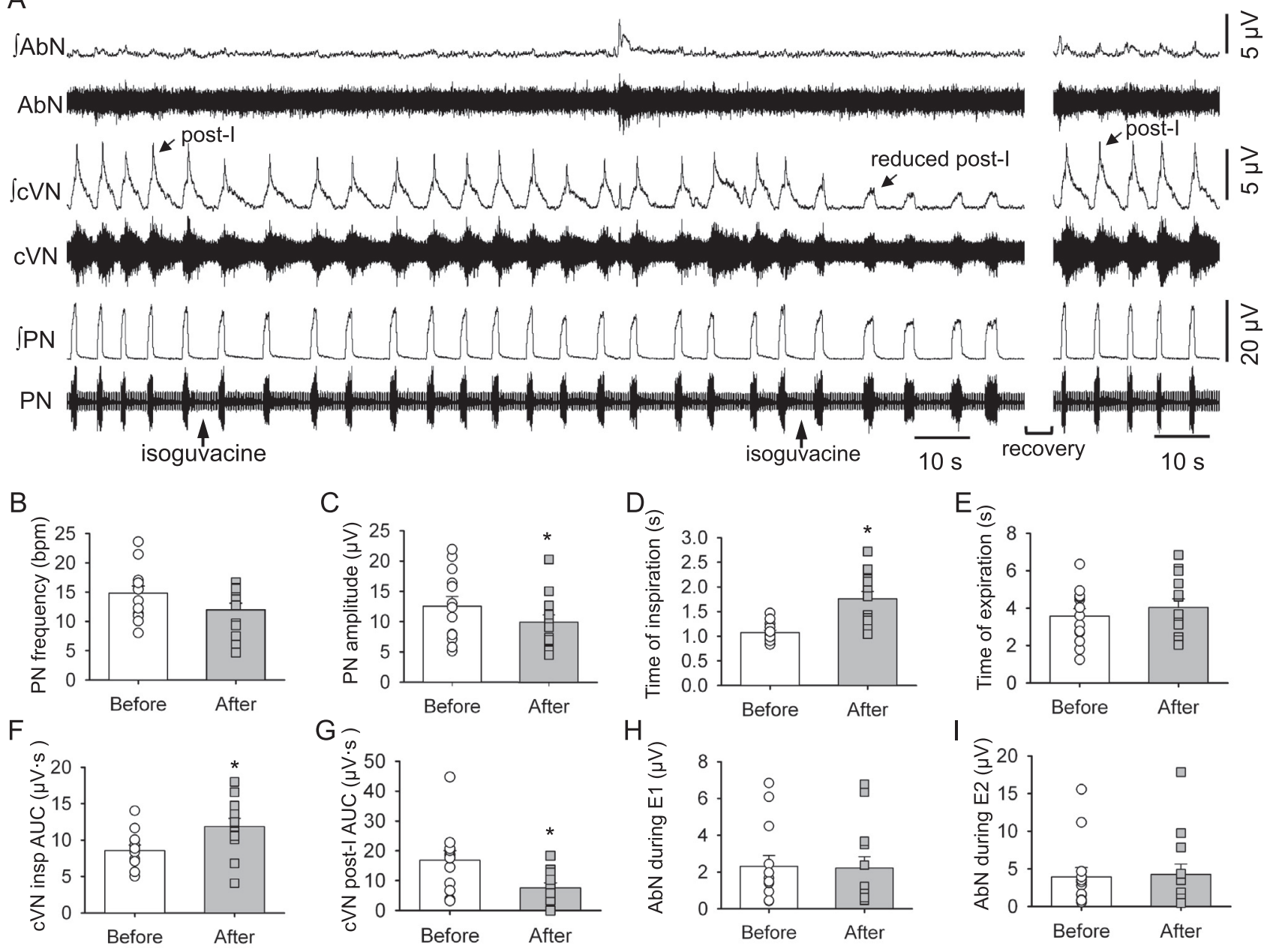

Fig. 3. Raw and integrated ( $\int$ ) recordings of the abdominal (AbN), cervical vagus (cVN), and phrenic nerve (PN) activities of an in situ preparation, representative of the group, showing the changes in baseline respiratory parameters after bilateral injections of the isoguvacine (arrows) into the KF. The two injections were completed within two minutes, and after the second injection there was a marked reduction in the cVN post-inspiratory (post-I) activity. These maximal effects lasted between 1 and $5 \mathrm{~min}$, after which there was a recovery of the cVN post-inspiratory activity. Average values are presented for PN burst frequency (B) and amplitude (C), time of inspiration (D) and expiration (E), cVN inspiratory (F) and postinspiratory $(\mathrm{G})$ area under the curve $(\mathrm{AUC})$ and $\mathrm{AbN}$ activity during $\mathrm{E} 1(\mathrm{H})$ and $\mathrm{E} 2(\mathrm{I})$ phases. Bars represent means $\pm \mathrm{S}$.E.M. $n=13$, * represents $\mathrm{P}<0.05$.

$p<0.001$, Fig. 4E) and evoked a marked increase in AbN activity $(\Delta: 86.31 \pm 11.98 \%, \quad F(1,12)=10.73$, $p=0.007$, Fig. $4 \mathrm{~F})$. The $\mathrm{AbN}$ response was initially characterized by the presence of peaks of activity during post-I and late-E phases, which decreased to only late-E bursts as $A b N$ discharge returned to the initial conditions.

Inhibition of the KF with isoguvacine microinjections notably modified the pattern of chemoreflex respiratory response to $\mathrm{KCN}$ (Fig. 4A, B). The effect of KF inhibition on the $\mathrm{KCN}$-induced tachypneic response was variable among experiments. The magnitude of reflex tachypnea remained unchanged in some preparations $(n=7$ Fig. 4A) whilst others showed a reduction in respiratory frequency in response to $\mathrm{KCN}(n=6$, Fig. 4B) compared to the control response. On average, the magnitude of the elevation in PN burst frequency before and after peripheral chemoreceptor stimulation was significantly reduced after $K F$ inhibition ( $\triangle P N$ burst frequency: $8.33 \pm 0.92$ vs. $2.12 \pm 2.28 \mathrm{bpm}, F(1,12)$ $=5.77, p=0.033$, Fig. $4 \mathrm{C})$. Irrespective of the pattern of change in PN burst frequency, KCN still evoked an increase in PN burst amplitude after KF inhibition $(\triangle \mathrm{PN}$ amp: $19.17 \pm 4.39$ vs. $28.52 \pm 4.26 \%, F(1,12)=2.91$, $p=0.114$, Fig. 4D). The increase in cVN inspiratory activity after $\mathrm{KCN}$ stimulation was maintained after $\mathrm{KF}$ inhibition $(\Delta: 14.34 \pm 1.68$ vs. $17.52 \pm 2.35 \%, F(1,11)$ $=3.00, p=0.111$, Fig. $4 \mathrm{E})$, but the increase in $\mathrm{cVN}$ post-I activity was significantly attenuated $(\Delta: 26.20$ \pm 4.19 vs. $7.65 \pm 2.81 \%, \quad F(1,11)=9.82, p=0.010$ Fig. 4E). Moreover, the amplitude of $\mathrm{AbN}$ response increased significantly $(\Delta: 86.31 \pm 11.98$ vs. 121.54 $\pm 14.49 \%, F(1,12)=11.19, p=0.006$, Fig. $4 \mathrm{~F})$ after $\mathrm{KF}$ inhibition and was characterized by a large burst 
A

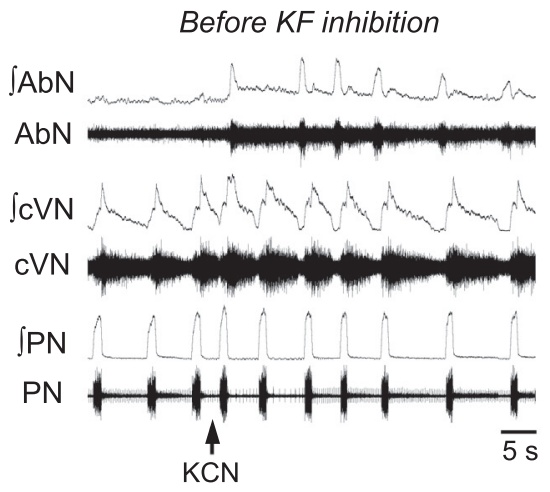

B

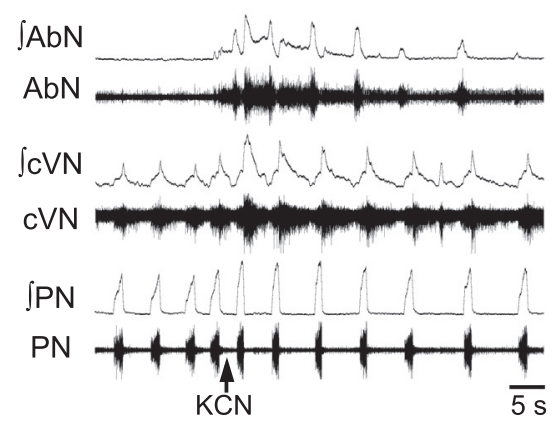

C

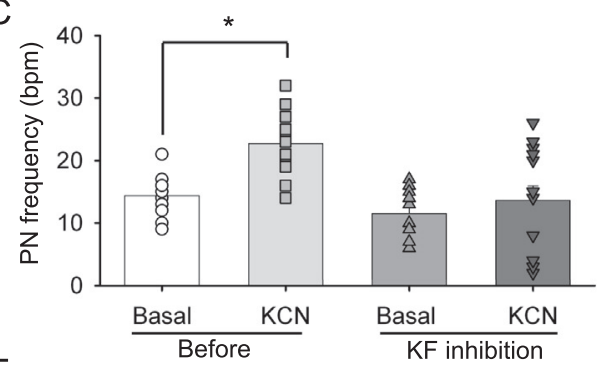

E

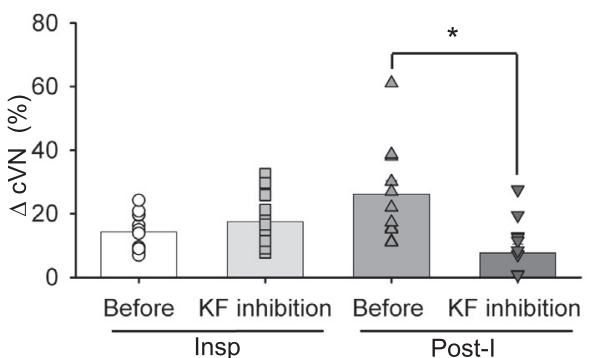

After KF inhibition

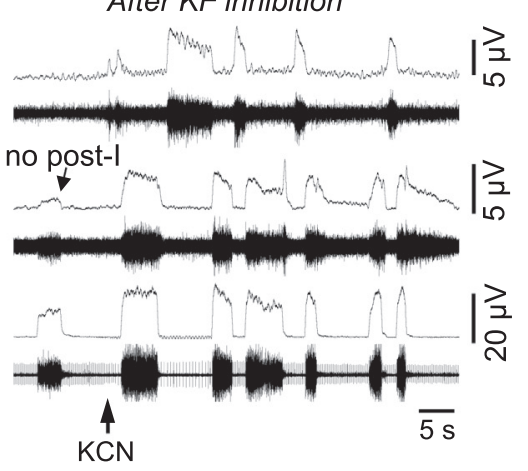

After KF inhibition
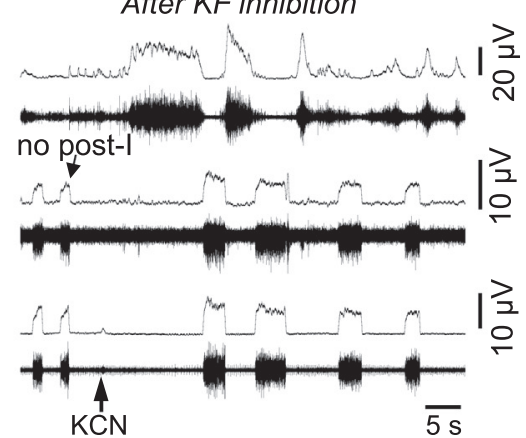

D

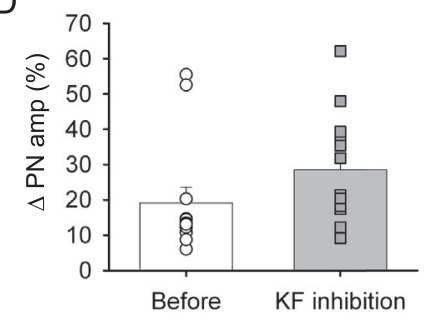

F

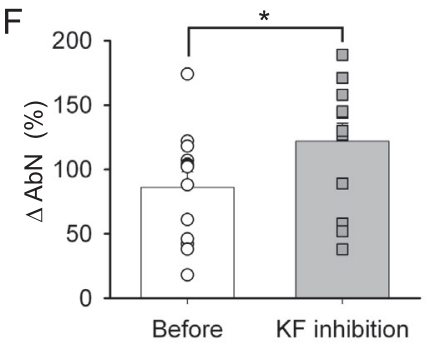

Fig. 4. Raw and integrated $\left(\int\right)$ recordings of abdominal $(A b N)$, cervical vagus (cVN) and phrenic nerve $(P N)$ activities of an in situ preparation, representative of the group, showing the increase in breathing frequency upon peripheral chemoreflex activation (with $\mathrm{KCN}$, arrows) before and after the inhibition of the KF with bilateral microinjections of isoguvacine (10 mM, ISO arrow) Representative traces of the reduced tachypnea observed in 7 rats upon KCN stimulation after kF inhibition $(\mathrm{A})$ and the bradypnea observed in 6 rats upon $\mathrm{KCN}$ stimulation after $\mathrm{KF}$ inhibition (B) are both shown. Average values are presented for PN burst frequency under basal conditions and after stimulation with $\mathrm{KCN}$ observed before KF inhibition and after KF inhibition (C), and for the percentage changes (relative to baseline) in PN burst amplitude (D), inspiratory and postinspiratory (post-I) cVN activities (E) and $\mathrm{AbN}$ amplitude (F) after KCN before and after KF inhibition. Bars represent means \pm S.E.M. $n=13,{ }^{*}$ represents $p<0.05$.

during the expiratory period (Fig. 4A, B). Note that following the initial $\mathrm{AbN}$ burst, subsequent $\mathrm{AbN}$ bursts displayed a decrementing pattern of discharge (Figs. 4A, B).

\section{Effect of KF inhibition with isoguvacine on the hypercapnic response}

Upon exposure to hypercapnia $(n=10$, Fig. 5A), there was a significant increase in the $A b N$ activity during the late- $E$ phase compared to baseline conditions $(\triangle \mathrm{AbN}: \quad 132.49 \pm 26.86 \%, \quad F(1,9)$ $=9.94, p=0.012)$. The emergence of these AbN late-E bursts was accompanied by: (i) no significant changes in PN burst frequency between baseline and hypercapnic conditions before $\mathrm{KF}$ inhibition $(15.30 \pm 1.76$ vs. $13.28 \pm 0.80 \mathrm{bpm}$, $\chi^{2}(3, \quad n=10)=16.76, \quad p=0.144$, Fig. 5B) or amplitude compared to baseline conditions ( $\triangle \mathrm{PN}$ amp: 6.49 $\pm 6.14 \%, \quad F(3,27)=1.11$, $p=0.363$, Fig. $5 \mathrm{D}$ ); (ii) a significant reduction in inspiratory $(1.06 \pm 0.08$ vs. $0.86 \pm 0.08 \mathrm{~s} ; \quad F(3,27)=13.33$, $p<0.001$, Fig. $5 C$ ) and expiratory times $(4.00 \pm 0.3$ vs. $3.1 \pm 0.2 \mathrm{~s} ; F$ $(3,27)=3.54, \quad p=0.028)$ between baseline and hypercapnic conditions before KF inhibition; and (iii) a significant increase in the cVN post-I peak $(\Delta c \mathrm{cVN}$ post-I peak: 25.34 $\pm 5.40 \% ; \quad F(1,8)=15.84$, $p=0.004$, Fig. $5 \mathrm{E})$ and a tendency to decrease the cVN post-I duration $(\triangle \mathrm{cVN}$ post-I duration: -0.58 $\pm 0.28 \mathrm{~s} ; F(1,8)=4.43, p=0.068)$.

The inhibition of the KF during hypercapnia caused a significant decrease in $\mathrm{PN}$ burst frequency compared to baseline conditions and hypercapnic conditions before KF inhibition $(10.48 \pm 0.68$ vs. 15.30 \pm 1.76 and $13.28 \pm 0.80 \mathrm{bpm} ; \chi^{2}(3$, $n=10)=16.76, \quad p<0.001$, Fig. 5B). This drop in PN burst frequency was associated with an increase in inspiratory time compared to baseline conditions and hypercapnic conditions before KF inhibition $(1.37 \pm 0.09$ vs. 1.06 \pm 0.08 and $0.86 \pm 0.08 \mathrm{~s}, F(3,27)$ $=13.33, \quad p<0.001, \quad$ Fig. $5 \mathrm{C}$ ). Inhibition of the KF had no significant effect on PN amplitude $(\triangle \mathrm{PN}$ amp: $-2 \pm 7 \%$; $F(2,18)=1.91$ $p=0.177$, Fig. 5D). Microinjections of isoguvacine in the KF significantly depressed $\mathrm{cVN}$ post-I activity $(\triangle \mathrm{cVN}$ post-I peak: $-78.49 \pm 6.34 \%, \quad \chi^{2}(2, \quad n=9)=14.00$, $p<0.001$, Fig. 5E). Interestingly, although the KF inhibition did not modify the amplitude of hypercapniainduced $\mathrm{AbN}$ activity $(\triangle \mathrm{AbN}$ late-E: $171.05 \pm 36.66 \%, F$ 
A

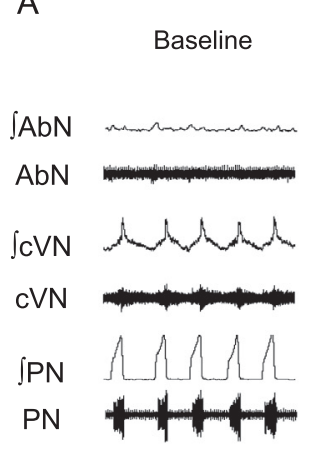

$\mathrm{B}$
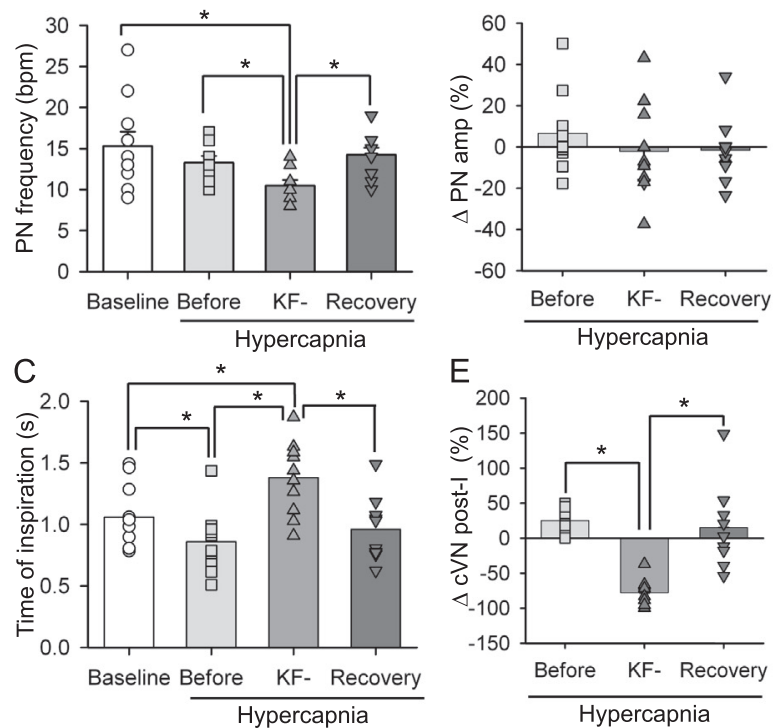

$$
\text { KF inhibition }
$$

Late-E onset
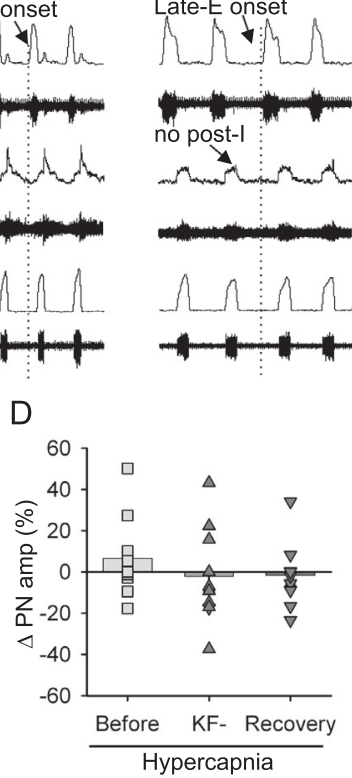

Hypercapnia $\left(8-10 \% \mathrm{CO}_{2}\right)$
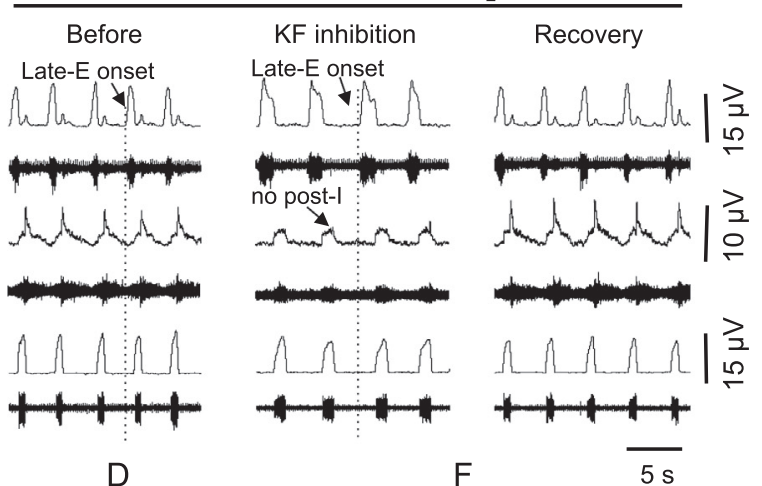

$\stackrel{\text { in }}{\frac{1}{2}}$

$\mathrm{F}$

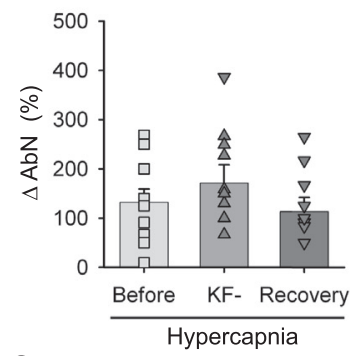

G

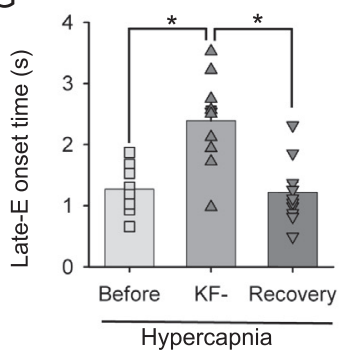

Fig. 5. Integrated $\left(\int\right)$ traces of abdominal $(A b N)$, cervical vagus (cVN) and phrenic nerve (PN) activities from an in situ preparation, representative of the group, showing the pattern of respiratory motor activities under baseline $\left(5 \% \mathrm{CO}_{2}, 95 \% \mathrm{O}_{2}\right)$ and hypercapnic conditions $\left(8 \% \mathrm{CO}_{2}, 92 \%\right.$ $\mathrm{CO}_{2}$ ) before, during $\mathrm{KF}$ inhibition with isoguvacine $(10 \mathrm{mM})$ and after recovery from the KF inhibition. Note that after KF inhibition, cVN post-I activity decreased and the onset of the AbN late$E$ burst increased. Average values are presented for PN burst frequency $(B)$ and the time of inspiration $(\mathrm{C})$ at baseline, during hypercapnic conditions before KF inhibition, during KF inhibition (KF-) and after recovery from KF inhibition (C), the percentage change (relative to baseline) in PN (D), cVN (E) and abdominal amplitudes (F) and the ABN onset (G) between baseline conditions and during hypercapnic conditions before KF inhibition, during KF inhibition (KF-) and after recovery from KF inhibition. Bars represent means \pm S.E.M. $n=10$, * represents $p<0.05$.

$(2,18)=2.31, p=0.128$, Fig. $5 F)$, the duration of the late-E bursts increased as a result of the earlier onset of late-E bursts relative to $\mathrm{PN}$ bursts $(2.39 \pm 0.74$ vs. 1.27 $\pm 0.36 \mathrm{~s}, F(2,18)=22.21, p<0.001$, Fig. $5 \mathrm{G})$. After the recovery from the inhibition of the KF, while still being exposed to hypercapnia, the $\mathrm{PN}, \mathrm{cVN}$ and $\mathrm{AbN}$ responses were comparable to control hypercapnic conditions (Fig. 5B-F).

\section{DISCUSSION}

Breathing is the product of the communication between multiple specialized neuronal networks within the brainstem. To date, most research conducted on the involvement of the pons in generating or modifying the breathing pattern has focused on its role in triggering the commencement of post-inspiratory (post-I) activity and the inspiratory off-switch (see Mörschel and Dutschmann, 2009 for review). Here we show that descending inputs from the pons, specifically the Kölliker-Fuse nucleus (KF), restrain the onset of late-E abdominal nerve activity and thus act as a timekeeper for the active expiratory pattern observed after stimulation of peripheral $\mathrm{O}_{2}$ chemoreceptors and under hypercapnic conditions. This highlights the role of the pons in coordinating active expiration (AE) and outlines the complexity and multifunctionality of the pontine and medullary centers in controlling breathing.

\section{The role of the KF in the control of the expiratory phase during eupnea}

It has been suggested that the threephase eupneic breathing pattern relies on an inhibitory ring comprised of the post-inspiratory (post-I) and augmenting-expiratory

(aug-E) neurons of the BötC and the inspiratory (I) neurons of the preBötC (Lindsey et al., 1987; Ezure and Manabe, 1988; Smith et al., 2007). The BötC post-I neurons inhibit I neurons to terminate inspiration (Rubin et al., 2011 for review). Subsequently, due to their intrinsic electrophysiological properties and increasing inhibition from the aug- $E$ neurons of the BötC, the post-I neurons stop firing producing the second phase of expiration (Rubin et al., 2011 for review). Finally, the early-I neurons of the preBötC fire, inhibiting all of the expiratory neurons of the BötC (see Rubin et al., 2011 for review). Although this inhibitory ring appears to be the kernel of central respiratory pattern and rhythm generation (Molkov et al., 2010), studies indicate that its function depends on synaptic interactions of BötC and preBötC with other respiratory compartments of the brainstem, especially the pons (Smith et al., 2007; Janczewski et al., 2013).

There is growing evidence to support the role of the pons in generating post-I activity. Dutschmann and Herbert (2006) found that pharmacological inhibition of the KF with isoguvacine decreased baseline breathing frequency, eliminated recurrent laryngeal nerve post-I activity and reduced upper airway resistance during expiration. Smith et al. (2007) and Abdala et al. (2009a) have also shown that complete transection of the pons, which causes a shift from a three-phase (I, post-I, E) to a twophase breathing cycle (I, E), eliminated the BötC post-I neuronal activity and the post-I component of cVN 
activity. Recently, Bautista and Dutschmann (2014) found that inhibition of the KF resulted in a significant decrease in hypoglossal motor activity during the inspiratory and post-I phases. There is evidence suggesting reciprocal inhibition of the post-I neurons with the aug-E neurons (Ezure et al., 2003; Smith et al., 2009), and the pons appears to play a role in coordinating the activity of these two neuronal groups. These studies provide overwhelming support of the role of the $\mathrm{KF}$ in acting as the inspiratory-off switch, providing tonic drive to excite the post-I neuron activity and thus the termination of I neuron activity, contributing to the functioning of the BötC/preBötC inhibitory ring.

From our data, inhibition of the KF decreased cVN post-I activity and increased inspiratory motor activity (duration and amplitude), highlighting the importance of the KF in initiating the post-I activity and the transition from the I to post-I phase. Bautista and Dutschmann (2016) have shown that KF inhibition does not modify basal AbN activity, suggesting that the $\mathrm{KF}$ is not required for the transition from post-I to the second phase of expiration (E2) under eupneic conditions. In our study, we did not observe differences in baseline AbN activity before or after inhibition of the KF, providing further support to previous observation that the KF is not essential for the transition from post-I to E2 during eupnea - an event that must rely mainly on intrinsic properties of adapting postI neurons of the BötC (Paton, 1996; Molkov et al., 2010).

\section{The role of the KF in the control of late-E activity during peripheral and central chemoreflex activation}

Under eupneic conditions, tonic abdominal expiratory activity is low and expiratory flow is initiated passively by recoil forces of the lungs and the chest (Jenkin and Milsom, 2014). Of the studies that have investigated active expiration (AE) with increasing respiratory drive, $A E$ typically appears at the end of the expiratory (late-E) pause (Sherrey et al., 1988; Taccola et al., 2007; Abdala et al., 2009a,b; Pagliardini et al., 2011). With greater respiratory drive, AE begins earlier, occupying more of the expiratory phase (Gautier et al., 1973; Sherrey et al., 1988; De Troyer et al., 1989). The generation of late-E abdominal activity depends on the activation of the RTN/pFRG (Janczewski and Feldman, 2006), which contains expiratory neurons that are silenced under resting conditions due to tonic inhibitory drive (Pagliardini et al., 2011). Under hypoxic or hypercapnic conditions, it has been suggested that the RTN/pFRG late-E activity emerges due to increased excitatory and reduced inhibitory drives to the RTN/pFRG (Abdala et al., 2009a,b; Marina et al., 2010; Moraes et al., 2012a,b) and provides excitatory inputs to the ventral respiratory column (Molkov et al., 2010).

Peripheral chemoreceptor stimulation by KCN evoked an increase in respiratory frequency, vagal post-I activity, abdominal post-I and abdominal late-E motor activities. When the KF was inhibited, the increase in vagal post-I activity was largely depressed and the abdominal response was potentiated and occupied the entire expiratory phase. Given that the post-I neurons of the
BötC are considered a source of inhibition of the RTN/ pFRG (Abdala et al., 2009a,b), we conclude that $\mathrm{O}_{2}$ chemoreflex-induced post-I activity restrains the emergence of late-E activity, which emerges only during the end of the expiratory period (when post-I activity decreases). With the inhibition of the KF, late-E activity was released from inhibition (consequent to post-I depression) and the excitatory inputs, potentially from the NTS (Takakura et al., 2006; Moraes et al., 2012a,b), generated an amplified abdominal response. We also observed that the tachypneic response to $\mathrm{KCN}$, on average, was also attenuated after KF inhibition. Importantly, the variability that we observed regarding the phrenic burst frequency response to $\mathrm{KCN}$ after isoguvacine was not related to any obvious differences in the microinjection site location within the KF. However, we noticed a temporal relationship between the magnitude of tachypnea and the emergence of the abdominal response to KCN. Based on our results, the abdominal response appears before the phrenic excitation as an initial expiratory lengthening preceding the increase in phrenic burst frequency (see Fig. 4A,B) - which may be related to the time of stimulation of peripheral chemoreceptors within the respiratory cycle (during I or E phase). Considering that the recruitment of RTN/pFRG late-E neurons is associated with enhanced BötC aug-E neuron activity (Abdala et al., 2009a), the augmented late-E neuron activation after KF inhibition may also contribute to the observed expiratory lengthening during peripheral chemoreceptor stimulation, preventing the tachypneic reflex response. Our data provide new evidence that the KF regulates the post-I activity during peripheral chemoreflex activation, which controls the duration of abdominal late- $E$ activity and the magnitude of the increase in respiratory frequency.

Under hypercapnic conditions, abdominal activity appears to emerge as a result of RTN/pFRG late-E neuronal activation (Janczewski and Feldman, 2006; Abdala et al., 2009a,b; Molkov et al., 2010) due to the stimulation of $\mathrm{CO}_{2}$-chemosensitive neurons (Marina et al., 2010). Interestingly, post-I expiratory breaking activity is always conserved in the breathing cycle, and thus late-E activity is always preceded by post-I activity (Sherrey et al., 1988; Mörschel and Dutschmann, 2009). This suggests that the transition from passive to active expiration is likely controlled by the termination of post-I neuron activity. Abdala et al. (2009) demonstrated that the abdominal late-E bursts during hypercapnia $(10 \%$ $\mathrm{CO}_{2}$ ) occur in parallel with shortening of vagal post-I activity. Our results show that vagal post-I duration decreases, albeit not significantly, with the presence of late-E $A E$. The lack of significance is probably due to the use of lower $\mathrm{CO}_{2}$ levels $\left(8 \% \mathrm{CO}_{2}\right)$ compared to previous studies (Abdala et al., 2009a). When the KF was inhibited, post-I activity was further depressed and $\mathrm{AbN}$ late-E activity was amplified. Consequently, expiratory time was prolonged, potentially by increased activity of BötC aug-E neurons (see Abdala et al., 2009a) and respiratory frequency decreased. In conjunction, our data support that the KFdependent post-I neuronal activity inhibits the mechanisms generating $A E$ in hypercapnia. Additional studies are still required to identify how KF neurons interact with 
the neural network involved with the generation of active expiration. However, two new hypotheses are raised: 1) the KF may be inhibited during hypercapnia, causing depression of tonic drive to BötC post-I neurons; or 2) the BötC may be inhibited during hypercapnia, and KF tonic drive may maintain post-I activity during this period of inhibition.

\section{CONCLUSION}

The pons, specifically the $\mathrm{KF}$, is considered to be the inspiratory off-switch, triggering the start of post-I neuron activity and consequent inhibition of inspiratory neuronal activity. Our study concludes that the KF also plays a role in inhibiting the emergence of the late- $E$ abdominal activity under conditions of metabolic challenge, acting as a timekeeper for active expiration. While the mechanisms that allow the pons to control the duration of late- $\mathrm{E} A b N$ activity remain unknown, it is clear that the KF is critical in determining the duration of late- $E$ activity observed during hypercapnia and hypoxia. Our data extend the current knowledge about the respiratory central pattern generator and open new possibilities to help the development of cardiorespiratory changes associated with pathological states of central and peripheral chemoreceptor hyperactivity, such as sleep breathing disorders (Barnett et al., 2017), neurogenic hypertension (Moraes et al. 2013, 2014a) and conditions of chronic hypoxia (Moraes et al., 2014b).

Acknowledgements-This work was supported by São Paulo Research Foundation - Brazil (FAPESP, grant 2013/17251-6) and by NIH (grant R01 AT008632), and by a grant from the NSERC of Canada to WKM. SEMJ, WKM and DBZ designed the experimental protocols. SEMJ performed the experiments. SEMJ and DBZ analyzed the data. SEMJ, WKM and DBZ interpreted the data, drafted, revised and approved the final version of the manuscript.

\section{REFERENCES}

Abdala APL, Rybak IA, Smith JC, Paton JFR (2009a) Abdominal expiratory activity in the rat brainstem-spinal cord in situ: patterns, origins and implications for respiratory rhythm generation. J Physiol 587:3539-3559.

Abdala AP, Dutschmann M, Bissonnette JM, Paton JF (2009b) Correction of respiratory disorders in a mouse model of Rett syndrome. Proc Natl Acad Sci USA 107:18208-182013.

Abraham KA, Feingold H, Fuller DD, Jenkins M, Mateika JH, Fregosi RF (2002) Respiratory-related activation of human abdominal muscles during exercise. J Physiol 541:653-663.

Alheid GF, McCrimmon DR (2008) The chemical neuroanatomy of breathing. Respir Physiol Neurobiol 164:3-11.

Bautista TG, Dutschmann M (2014) Inhibition of the pontine köllikerFuse nucleus abolishes eupneic inspiratory hypoglossal motor discharge in the rat. Neuroscience 267:22-29.

Bautista TG, Dutschmann M (2016) The role of the Kölliker-Fuse nucleus in the determination abdominal motor output in a perfused brainstem preparation of juvenile rat. Respir Physiol Neurobiol 226:102-109.

Barnett WH, Abdala AP, Paton JF, Rybak IA, Zoccal DB, Molkov YI (2017) Chemoreception and neuroplasticity in respiratory circuits. Exp Neurol 287:153-164.
Davis GM, Bureau MA (1987) Pulmonary and chest wall mechanics in the control of respiration in the newborn. Clin Perinatol 14:551-579.

De Troyer A, Gilmartin JJ, Ninane V (1989) Abdominal muscle use during breathing in unanesthetized dogs. J Appl Physiol 66:20-27.

Dutschmann M, Herbert H (2006) The Kölliker-Fuse nucleus gates the post-Inspiratory phase of the respiratory cycle to control inspiratory off-switch and upper airway resistance in rat. Eur $\mathrm{J}$ Neurosci 24:1071-1084.

Dutschmann M, Jones SE, Subramanian HH, Stanic D, Bautista TG (2014) The physiological significance of postinspiration in respiratory control. Prog Brain Res 212:113-130.

Ezure K, Manabe M (1988) Decrementing expiratory neurons of the Bötzinger complex. Exp Brain Res 72:159-166.

Ezure K, Tanaka I, Kondo M (2003) Glycine is used as a transmitter by decrementing expiratory neurons of the ventrolateral medulla in the rat. J Neurosci 23:8941-8948.

Feldman JL, McCrimmon DR (2003) Neural control of breathing. In: Squire L, Bloom FE, Spitzer NC, Squire LR, Berg D, du Lac Sasha, Ghosh A, editors. Fundamental neuroscience. Boston, MA: Academic Press. p. 967-990.

Feldman JL, Del Negro CA, Gray PA (2013) Understanding the rhythm of breathing: so near yet so far. Annu Rev Physiol 75:423-452.

Gautier H, Remmers JE, Bartlett Jr D (1973) Control of the duration of expiration. Resp Physiol 18:205-221.

Janczewski WA, Feldman JL (2006) Distinct rhythm generators for inspiration and expiration in the juvenile rat. $J$ Physiol 570:407-420.

Janczewski WA, Tashima A, Hsu P, Cui Y, Feldman JL (2013) Role of inhibition in respiratory pattern generation. J Neurosci 33:5454-5465.

Jenkin SE, Milsom WK (2014) Expiration: breathing's other face. Prog Brain Res 212:131-147.

Jones SE, Dutschmann M (2016) Testing the hypothesis of neurodegeneracy in respiratory network function with a priori transected arterially perfused brain stem preparation of rat. $J$ Neurophysiol 115:2593-2607.

Lemes EV, Zoccal DB (2014) Vagal afferent control of abdominal expiratory activity in response to hypoxia and hypercapnia in rats. Respir Physiol Neurobiol 203:90-97.

Lindsey BG, Segers LS, Shannon R (1987) Functional associations among simultaneously monitored lateral medullary respiratory neurons in the cat. II. Evidence for inhibitory actions of expiratory neurons. J Neurophysiol 57:1101-1117.

Marina N, Abdala AP, Trapp S, Li A, Nattie EE (2010) Essential role of Phox $2 \mathrm{~b}$-expressing ventrolateral brainstem neurons in the chemosensory control of inspiration and expiration. J Neurosci 30:12466-12473.

Molkov YI, Abdala AP, Bacak BJ, Smith JC, Paton JF, Rybak IA (2010) Late-expiratory activity: emergence and interactions with the respiratory CpG. J Neurophysiol 104:2713-2729.

Moraes DJ, Dias MB, Cavalcanti-Kwiatkoski R, Machado BH, Zoccal DB (2012a) Contribution of the retrotrapezoid nucleus/parafacial respiratory region to the expiratory-sympathetic coupling in response to peripheral chemoreflex in rats. J Neurophysiol 108:882-890.

Moraes DJ, Zoccal DB, Machado BH (2012b) Medullary respiratory network drives sympathetic overactivity and hypertension in rats submitted to chronic intermittent hypoxia. Hypertension 60:1374-1380.

Moraes DJ, Machado BH, Paton JF (2014a) Specific respiratory neuron types have increased excitability that drive presympathetic neurones in neurogenic hypertension. Hypertension 63:1309-1318.

Moraes DJ, Bonagamba LG, Costa KM, Costa-Silva JH, Zoccal DB, Machado BH (2014b) Short-term sustained hypoxia induces changes in the coupling of sympathetic and respiratory activities in rats. J Physiol 592:2013-2033. 
Moraes DJ, da Silva MP, Bonagamba LG, Mecawi AS, Zoccal DB, Antunes-Rodrigues J, Varanda WA, Machado $\mathrm{BH}$ (2013) Electrophysiological properties of rostral ventrolateral medulla presympathetic neurons modulated by the respiratory network in rats. J Neurosci 33:19223-19237.

Mörschel M, Dutschmann M (2009) Pontine respiratory activity involved in inspiratory/expiratory phase transition. Philos Trans R Soc Lond Biol Sci 364:2517-2526.

Onimaru H, Homma I (2003) A novel functional neuron group for respiratory rhythm generation in the ventral medulla. J Neurosci 23:1478-1486.

Pagliardini S, Janczewski WA, Tan W, Dickson CT, Deisseroth K, Feldman JL (2011) Active expiration induced by excitation of ventral medulla in adult anesthetized rats. $J$ Neurosci 31:2895-2905.

Paton JF (1996) A working heart-brainstem preparation of the mouse. J Neurosci Methods 65:63-68.

Paxinos G, Watson C (1998) The rat brain in stereotaxic coordinates. 6th ed. London: Academic Press.

Richter DW (1982) Generation and maintenance of the respiratory rhythm. J Exp Biol 100:93-107.

Richter DW (1996) Neural regulation of respiration: rhythmogenesis and afferent control. In: Gregor R, Windhorst $U$, editors. Comprehensive human physiology, vol. II. Berlin, Germany: Springer-Verlag. p. 2079-2095.

Rosin DL, Chang DA, Guyenet PG (2006) Afferent and efferent connections of the rat retrotrapezoid nucleus. J Comp Neurol 2499:64-89.

Rubin JE, Bartholomew JB, Molkov YI, Shevtsova NA, Smith JC, Rybak IA (2011) Interacting oscillations in neural control of breathing: modeling and qualitative analysis. J Comput Neurosci 30:607-632.
Sherrey JH, Pollard MJ, Megirian D (1988) Proprioceptive, chemoreceptive and sleep state modulation of expiratory muscle activity in the rat. Exp Neurol 101:50-62.

Silva JN, Tanabe FM, Moeira TS, Takakura AC (2016) Neuroanatomical and physiological evidence that the retrotrapezoid nucleus/parafacial region regulates expiration in adult rats. Respir Physiol Neurobiol 227:9-22.

Smith JC, Ellenberger H, Ballanyi K, Richter DW, Feldman JL (1991) Pre-Bötzinger complex: brainstem region that may generate respiratory rhythm in mammals. Science 254:726-729.

Smith JC, Abdala AP, Koizumi H, Rybak IA, Paton JF (2007) Spatial and functional architecture of the mammalian brain stem respiratory network: a hierarchy of three oscillatory mechanisms. J Neurophysiol 98:3370-3387.

Smith JC, Abdala APL, Rybak IA, Paton JFR (2009) Structural and functional architecture of respiratory networks in the mammalian brainstem. Phil Trans R Soc B 364:2577-2587.

Smith JC, Abdala APL, Borgmann A, Rybak IA, Paton JFR (2013) Brainstem respiratory networks: building blocks and microcircuits. Trends Neurosci 36:152-162.

Taccola G, Secchia L, Ballanyi K (2007) Anoxic persistence of lumbar respiratory bursts and block of lumbar locomotion in newborn rat brainstem-spinal cords. J Physiol 585:507-524.

Takakura AC, Moreira TS, Colombari E, West GH, Stornetta RL, Guyenet PG (2006) Peripheral chemoreceptor inputs to retrotrapezoid nucleus (RTN) $\mathrm{CO}_{2}$-sensitive neurons in rats. J Physiol 572:503-523.

Zoccal DB, Simms AE, Bonagamba LGH, Braga VA, Pickering AE, Paton JFR, Machado BH (2008) Increased sympathetic outflow in juvenile rats submitted to chronic intermittent hypoxia correlates with enhanced expiratory activity. J Physiol 586:3253-3265. 